\title{
UNE ÉPIDÉMIE PROVOQUÉE PAR LE « POU DES POISSONS » (ICHTHYOPHTIRIUS MULTIFILIIS FOUQUET) \\ SUR DES ALEVINS ET DES JEUNES TRUITES
}

\author{
Par H. BARTHÉLÉMY
}

Les recherches et les publications sur Ichthyophtirius multifiliis sont relativement nombreuses; la bibliographie en est longue. Malheureusement la plupart des cas signalés se rapportent à des infections d'aquarium de laboratoire, les observations dans la nature étant rares. Le manque de précision dans l'expérimentation très défectueuse font que la biologie du " pou des poissons ", les conditions et le mode de propagation, les mesures prophylactiques ou curatives à prendre pour le combattre sont encore assez obscures. Ces diverses considérations m'ont déterminé à publier cette note.

\section{Apparition de l'épidémie}

En juillet 1923, j’étais appêlé pour étudier une épidémie de nature inconnue sévissant sur des alevins et des jeunes truites de 5 à $7 \mathrm{~cm}$. de longueur d'un vaste établissement de pisciculture de l'Est de la France. Journellement les jeunes poissons périssaient par centaines ; il s'agissait avant tout d'enrayer la maladie qui prenait de plus en plus d'extension et risquait de faire péricliter une exploitation à plein rendement. Malgré l'abondance des matériaux mis à ma disposition, trop occupé par d'autres recherches biologiques, je n'ai pu ni voulu reprendre l'étude du cycle évolutif du parasite, sujet traité déjà par de nombreux auteurs. Je me contenterai done de signaler les observations et les expériences faites sur place ainsi que les procédés utilisés pour annihiler l'épidémie.

Pour mémoire, je rappellerai que fin juin et début de juillet 1923 , dans l'Est de la France, nous subissions de très fortes chaleurs. Des renseignements fournis par le pisciculteur, il résulte que l'épizootie a fait son apparition avec les grandes chaleurs, vers les 23-24 juin, puis elle augmentait d'intensité avec l'élévation de température du début de juillet. Fait curieux, qui a son importance, seuls les alevins de truite de rivière (Salmo fario) de provenance danoise périssent ; 
les autres espèces de truites, même les truites de rivière d'origine locale, résistent et ne paraissent pas malades. Les poissons atteints, entraînés sans doute par le courant qu'ils ne peuvent remonter, viennent mourir surtout à la sortie des bassins. Néanmoins on trouve encore de nombreux cadavres sur le pourtour des étangs, en particulier dans les " points mọts ", c'est-à-dire là où l'eau est peu renouvelée.

Avant de périr, les truites parasitées manquent de vivacité, leurs mouvements sont lents, elles nagent parfois en zig-zag, se retournent, puis piquent vers le fond du vivier et meurent. Tous symptô-

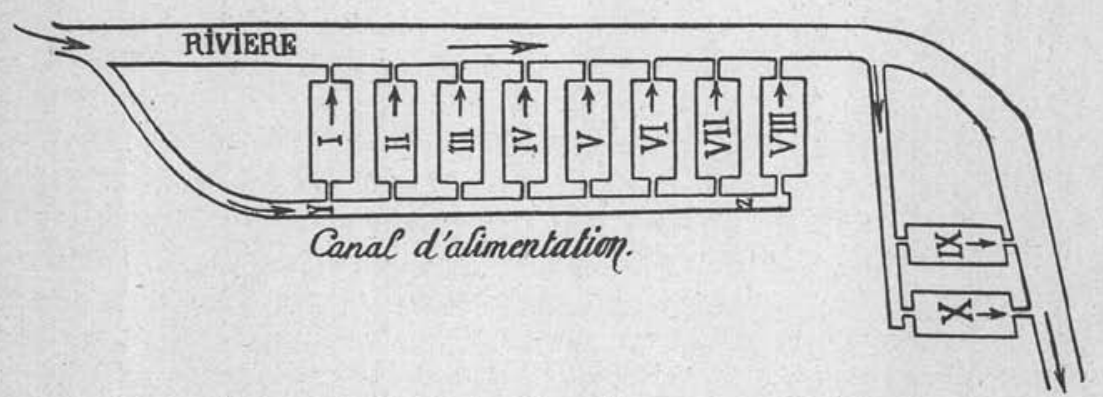

F1G. - Schéma représentant la disposition et le mode d'alimentation en eau des bassins de l'exploitation.

mes rappelant la maladie du vertige des poissons. L'infection est toute autre comme on le verra par la suite. Le pisciculteur signale également qu'en fin de journée les morts sont plus nombreux que le matin.

Je joins à cette note un schéma (fig.) représentant la disposition et le mode d'alimentation en eau des bassins de l'exploitation. Les élevages comprennent les espèces suivantes : truites arc-en-ciel (Salmo irideus) et truites de rivières (Salmo fario), d'origine locale ou d'origine danoise. En tous cas, chaque vivier isolé du voisin ne renferme qu'une seule espèce de poissons.

Le 13 juillet à 8 heures du matin la température de l'eau est notée dans les différents étangs. Le thermomètre centigrade marque $18^{\circ}, 5$ à l'arrivée d'eau en IX, $19^{\circ}$ à la sortie, $-19^{\circ}$ à la sortie de $\mathrm{X}$, $19^{\circ}$ à l'évacuation des bassins I, II, III, IV, V, VI, VIII, - et $20^{\circ}$ pour l'évacuation de VII. L'eau de la rivière ainsi que celle du canal d'alimentation en $\mathrm{Y}$ et en $\mathrm{Z}$ a une température de $17^{\circ}, 5$. 


\section{Etude sur place de l'évolution de l'épidémie}

Le 19 juillet, je me rends à l'établissement de pisciculture. L'étang VII renfermant des truites arc-en-ciel est infecté depuis quelques jours. Le vivier IX qui contient les malades du début de l'épidémie (truites de rivière d'origine danoise de provenance du bassin III) est en meilleur état; dans ce lot, la maladie paraît en décroissance. J'attribue ce résultat aux causes suivantes : $1^{\circ}$ alimentation en eau fournie plus abondamment dans cet étang; $2^{\circ}$ par suite d'orages successifs des journées précédentes, température plus basse de l'atmosphère et des eaux.

Dans les angles des bassins où l'eau se renouvelle peu ou pas, les truites atteintes sont encore abondantes ; elles se comportent comme des poissons soumis aux conditions asphyxiques et périssent en manifestant tous les caractères de la mort par asphyxie.

Si l'on recueille des cadavres d'alevins ayant cessé de vivre depuis plusieurs heures, l'autopsie ne décèle rien de particulier ni dans les différents organes (intestin, foie, rate, reins, muscles, cœur, cerveau) ni sur la peau. Sur les truites malades, au contraire, l'attention est attirée de suite par la présence de très nombreux petits points blancs visibles à l'oeil nu, de $200 \mu$ à $1 \mathrm{~mm}$. de diamètre, se trouvant sur la peau et les branchies. L'examen microscopique les identifie à Ichthyophtirius multifiliis, infusoires ciliés holotriches, sur la description desquels je n'entrerai pas, les auteurs l'ayant faite suffisamment. L'abondance de ces parasites sur les branchies explique la mort par asphyxie des alevins atteints ; les lésions de la peau étant insuffisantes pour déterminer des accidents graves. Sur les centaines de cadavres ou de poissons malades que j'ai examinés, je n'en ai vu aucun dont " les nageoires perdant leur peau se désagrègent de sorte que les arètes sortent librement " (Bruno Hofer). Le spécialiste munichois ne signale pas la présence des parasites sur l'appareil respiratoire ; il n'a vu dans la maladie qu'une affection de la peau. Neresheimer se montre plus explicite : "Le fait est déjà noté par Fouquet, Weltner et Doflein, que l'Ichthyophtirius se trouve sur les branchies. Mais ce fait d'après mon opinion est trop peu remarqué et n'a l'air que d'une exception amenée par le hasard. Moi-même dans tous les cas d'infection forte et moyenne je n'ai pas vu un seul individu dont les branchies ne soient libres de parasites. Très souvent je trouvais les branchies plus infectées que la peau. D'après ce qui est dit antérieurement, je ne doute pas que la majeure partie de l'action du parasite sur l'hôte est due à cette maladie des branchies " (Neresheimer). 
Soulignons dès à présent la disparition des parasites sur les cadavres des jeunes truites. Buschkiel a fait des observations analogues sur des poissons autres que la truite, morts en aquarium et parasités par Ichthyophtirius. - Ici le " pou des poissons" semble se comporter comme les poux et les puces des mammifères et des oiseaux. Dans l'un et l'autre cas, protozoaires, ou aptères, ou aphaniptères émigrent dès que l'hôte a cessé de vivre.

\section{Traitement de l'épidémie}

Ainsi que je l'annonçais au début de cette note, mon intervention avait été demandée pour déterminer la nature de la maladie et surtout pour l'enrayer si possible.

Les auteurs qui se sont occupés de cette question pratique sont peu encourageants. D'après Bruno Hofer, aucun produit chimique ne débarrasse efficacement l'hôte de ses parasites, parce que, dit-il, les infusoires se trouvent trop profondément enfoncés dans la peau du poisson. La raison est toute autre : le mucus abondant recouvrant les malades forme un enduit protecteur qui empêche la pénétration des réactifs chimiques. Neresheimer et Stiles ont essayé de guérir les poissons, mais ils n'ont pas réussi à détruire les parasites sans faire mourir l'hôte. Stiles tuait les Ichthyophtirius isolés par l'éosine, le bleu de méthylène et le sel de cuisine. Pour cet auteur, le meilleur moyen consiste à faire dans les aquariums des couches d'eau douce et d'eau salée dans lesquelles le parasite périclite. C'est un procédé bien peu pratique pour les vastes établissements de pisciculture.

Voyons ce que les différents zoologues écrivent sur la biologie du parasite. Pour Fouquet, "l'élévation de la température et l'abondance de l'eau paraissent favoriser le développement de la maladie. La présence de cailloux au fond de l'eau, en permettant aux poissons de se frotter pour détacher les parasites, contribue au contraire à atténuer la gravité du mal ". Le savant français essayant d'infecter des jeunes truites saines n'y parvient pas, mais il n'indique pas son protocole expérimental.

Buschkiel est plus précis. Il constate que la température semble jouer un grand rôle dans le développement de l'Ichthyophtirius : ainsi à la température moyenne de $17^{\circ}$, après 10 jours, on voyait les parasites à l'œil nu, et après 14-15 jours ils étaient assez grands pour être reconnus à un demi-mètre de distance. A $21^{\circ}$ il a pu voir de grands Ichthyophtirius après 8 jours ; une température de $13^{\circ}-14^{\circ}$ retarde le développement. Dans la plupart des cas, les parasites ne 
supportant pas l'agitation dans un verre de montre périssent rapidement.

Les cultures de Neresheimer périclitaient quand il les mettait dans l'eau froide. Enfin Fouquet, Buschkiel, Stiles, Kerbert, Neresheimer, Roth, tous sont d'accord pour reconnaitre que les infections ont lieu au printemps et en été. " Par contre, il semble prouvé que cette apparition périodique n'est pas une suite immédiate de la température, car des infections d'Ichthyophtirius ont été observées à des températures différentes. Kerbert indique que dans son aquarium, la température de l'eau en automne au moment où la culture a péri n'était pas moins élevée qu'au printemps, c'est-à-dire $17^{\circ}-18^{\circ}$ ” (Buschkiel). Plus loin, Buschkiel signale que le parasite peut être transporté directement par des mollusques aquatiques. Il a vu des Ichthyophtirius enkystés sur des coquilles de planorbes, mais il n'a jamais trouvé de parasites dans les parties molles du gastéropode. Dans les étangs contaminés que j’ai observés, les limnés étaient abondantes et semblaient se tenir de préférence dans le voisinage ou même sur les cadavres des poissons.

Avant mon intervention, le pisciculteur croyant à une infection microbienne avait introduit d'assez fortes doses de permanganate de potassium dans l'eau des bassins contaminés. L'effet avait été nul. Prélevant dans des tubes quelques échantillons de truites malades, j'avais remarqué que par frottement le poisson perd facilement et rapidement un grand nombre d'Ichthyophtirius qui ne tardent pas à tomber au fond du récipient. C'est le procédé employé par Neresheimer pour obtenir quelques kystes du parasite. Par ailleurs, des alevins parasités mis en aquarium en eau très courante et fraîche m'avaient montré que les jeunes truites se débarrassent relativement vite de leurs " poux " qui sont entraînés par le courant ou tombent au fond du bassin. Bruno Hofer signale un procédé analogue et recommande de maintenir les poissons malades quelque temps dans un courant d'eau très fort. D'autre part, comme je l'ai indiqué précédemment, les décès sont plus nombreux en fin de journée que le matin ; à la suite d'orages successifs, le débit d'eau plus fort et la température plus basse avaient provoqué une amélioration notable des premiers malades reportés dans l'étang IX.

Voilà un ensemble de faits à débrouiller, à interpréter et à utiliser. Les observations précédentes unilatérales se rapportent uniquement au parasite. L'attention n'est pas suffisamment attirée sur l'hôte. Cependant en parasitologie comme en bactériologie il faut tenir compte à la fois du parasite (animal, plante ou microbe) et de son habitat. Nul ne contredira que ce dernier facteur joue un rôle important dans le développement de la maladie. En général, plus l'hôte est 
résistant, moins le parasite a de prise et vice-versa. Les pisciculteurs n'ignorent pas, et l'excellent " Atlas de poche des poissons d'eau douce de France de C. Raveret-Wattel " leur rappelle que la truite commune ou truite de rivière (Salmo fario) " ne se plaît que dans les eaux fraîches et bien aérées ". Quant à la truite arc-en-ciel (Salmo irideus), l'auteur précité fait observer que : " tandis qu'on ne saurait cultiver la truite commune dans des eaux susceptibles de s'échauffer en été à plus de $18^{\circ}$, la truite arc-en-ciel peut supporter jusqu'à $25^{\circ}$ ou $26^{\circ}$. " Elle (la truite arc-en-ciel) possède une qualité qui la fera en toute circonstance apprécier des éleveurs : c'est sa rusticité à l'état d'alevins. Beaucoup plus robuste dès le tout jeune âge que la truite commune, elle n'est pas sujette, comme celle-ci, à une foule de maladies qui causent des pertes souvent considérables, même dans les élevages les mieux conduits. "

Au début de cette note, j'ai fait remarquer que l'épidémie avait débuté avec l'apparition de fortes chaleurs anormales pour de jeunes truites de rivière supportant mal le séjour prolongé dans les eaux à $18^{\circ}$ et plus. De par leur origine du Danemark, pays plus froid que le nôtre, ces alevins se montraient encore plus sensibles à l'élévation de température. Aussi rien de surprenant si l'épidémie a commencé dans ce lot.

D'après toutes les observations et faits signalés antérieurement, j'étais amené tout naturellement à traiter les bassins contaminés de la façon suivante :

$1^{\circ}$ Fournir aux étangs le plus d'eau possible afin d'établir un courant rapide qui entraîne les parasites libres, " décape " les poissons malades, réduit les points morts et surtout en abaissant la température diminue la rapidité du développement du parasite tout en augmentant la vigueur, la vivacité et la résistance des poissons.

$2^{\circ}$ Pécher, quand c'est possible, les truites parasitées et les reporter dans un chenal où circule une eau courante et très fraîche.

Il va de soi que l'eau du chenal ne doit pas retourner dans un étang en exploitation qui serait rapidement contaminé. Je fais cette remarque qui a son importance théorique et pratique, parce que, peu de jours avant mon intervention, des truites couvertes d'Ichthyophtirius avaient été reportées dans un autre établissement de pisciculture dont les viviers communiquaient entre eux, provoquant ainsi un début d'épidémie rapidement enrayée par une eau courante et fraîche. Cet accident prouve surabondamment qu'un vecteur, mollusque ou autre animal, n'est pas indispensable pour la propagation de la maladie ; le parasite pouvant vivre isolé dans l'eau est transporté par le courant. 
Les mesures préconisées précédemment ne tardèrent pas à donner les résultats Ies plus heureux. Avant le 19 juillet, par jour le nombre des décès était de 500 et plus par bassin contaminé; lors d'une visite aux étangs, le 26 juillet, j'ai pu constater que le vivier $\mathrm{N}^{\circ}$ IX n'avait eu que 3 morts en 24 heures, le $\mathrm{N}^{\circ} \mathrm{X}, 15$ seulement, et l'épidémie qui la semaine auparavant avait tendance à se propager dans d'autres bassins était en voie de disparition. Peu de temps après, la maladie était complètement terminée et depuis août 1923, sur les millions de poissons élevés dans l'établissement, les Ichthyophtirius n'ont plus causé un seul décès.

\section{Expérimentation sur Ichthyophtirius}

En présence d'une abondance de matériaux vivants et tout en surveillant les effets des mesures préventives et curatives indiquées ci-dessus, je faisais sur place quelques essais expérimentaux sur des truites malades. Y aurait-il une substance chimique capable de tuer rapidement le parasite fixé au poisson sans nuire à ce dernier ? En tous cas c'était une occasion de vérifier la résistance de l'Ichthyophtirius aux différents agents physiques et chimiques et par là prévoir un procédé de désinfection pratique pour les instruments, les récipients et les appareils utilisés en pisciculture. Tous les essais furent effectués dans de l'eau de source à la température de $12^{\circ}-14^{\circ}$ et contrôlés par l'examen microscopique qui permettait de juger de la mort du parasite d'après l'absence de mouvement. En pleine canıagne, mon arsenal chimique étant très limité, on ne sera pas surpris si les produits utilisés furent peu nombreux et parfois bizarres. Néanmoins je signale les résultats pour ce qu'ils ont donné.

I. Permanganate de potassium. - a) Dans les dilutions à $1 / 100.000$, après 2 heures, les poissons vivent encore ; après 4 heures de séjour les jeunes truites meurent, mais les parasites sont encore vivants ; après 15 heures et demie, les gros Ichthyophtirius sont immobiles, mais les petits s'agitent encore.

b) Pour des concentrations plus fortes, 1/20.000, 1/50.000 pendant une demi-heure, les résultats furent les suivants : même dans 1/20.000 les parasites restent vivants, alors que les poissons périssent déjà au bout de 20 minutes.

II. Ghlorure de sodium. - a) Après 15 heures $1 / 2$ de séjour dans $\mathrm{NaCl}$ à 2 pour cent les poissons vivent encore, mais les parasites aussi.

b) Si l'on emploie une solution de $\mathrm{NaCl}$ à 6 pour cent, après une demi-heure de contact, les parasites sont encore mobiles alors que les jeunes truites sont déjà mortes après un quart d'heure de séjour. 
III. Chlorure mercurique. - Au bout de quelques minutes dans les solutions de $\mathrm{HgCl}^{2}$ à $1 / 1.000$, les alevins périssent ; mais après une demi-heure de séjour on trouve à côté d'Ichthyophtirius immobiles d'assez nombreux parasites en mouvement.

IV. Ghaux. - $1 \mathrm{kgr}$. de chaux vive est dilué avec 120 litres d'eau (solution initiale). Dans un mélange de $50 \mathrm{cc}$. de cette solution primitive avec 1 litre d'eau, les alevins meurent presque immédiatement, les parasites périssent après 15 heures de contact. - Le lait de chaux dissout très rapidement petits et gros parasites.

v. Eau de Javel (liqueur du commerce). - En 4 minutes, les poissons meurent dans une solution renfermant $10 \mathrm{cc}$. d'eau de Javelle par litre d'eau. Quant aux parasites, ils sont encore mobiles après une demi-heure de séjour dans le liquide qui renferme $20 \mathrm{cc}$. d'eau de Javelle par litre d'eau.

Quatre minutes d'action d'une solution contenant $60 \mathrm{cc}$. par litre ne détruit pas les Ichthyophtirius, mais 20 minutes de séjour dans le inême réactif les rendent immobiles.

VI. Formol du commerce. - La solution ıdu commerce employée aux dilutions de $2 / 1.000,5 / 1.000,1 / 100,2 / 100$ donne les résultats suivants :

Après un séjour d'une demi-heure dans la solution à 2 pour mille, de nombreux parasites sont encore mobiles. Ils sont immobilisés dans le même laps de temps dans les solutions à $1 / 200,1 / 100$, $2 / 100 ; 1$ minute dans la solution à 1 pour cent les immobilise et les noircit. Déjà en 6 ou 7 minutes les jeunes poissons périssent même dans la dilution à 2 pour mille.

VII. Toluène. - Une ou deux minutes de séjour dans des mélanges de $1 \mathrm{cc}$. de toluène pour 4 litres d'eau tuent les jeunes truites alors que " les poux des poissons " sont encore vivants après une demiheure dans un mélange deux fois plus concentré.

VIII. Eau oxygénée. - Une solution à 4 pour cent de $\mathrm{H}^{2} \mathrm{O}^{2}$ du commerce dans l'eau ne détruit pas les parasites en 35 minutes; les alevins périssent en 12 minutes.

IX. Thymol. - Comme on le sait, le thymol n'est pas soluble dans l'eau. Une assez grande quantité de thymol ajoutée à l'eau remuée ne tue pas les Ichthyophtirius en 4 heures, tandis que l'hôte meurt au bout d'une demi-heure.

X. Ghloral hydraté. - L'eau additionnée de chloral hydraté en proportions variables ne détruit ni les parasites ni les poissons après 7 heures de séjour. 
XI. Gamphre. - Les truites ne résistent que quelques heures dans l'eau contenant du camphre, les Ichthyophtirius étant encore mobiles après 7 heures.

XII. Pyridine. - En solution à $1 / 2.000$, la pyridine " décape " en partie les jeunes poissons qui restent en vie plus de 5 heures; les plus gros parasites semblent émigrer, mais le microscope décèle encore de nombreux petits infusoires très mobiles dans le râclage des téguments du poisson. Dans la concentration à $2 / 1.000$, les truites périssent en quelques minutes, les Ichthyophtirius restant en vie.

XIII. Gréosote. - Ce produit ne donne pas de meilleurs résultats. Une dilution dans l'eau à $1 / 2.000$ tue les poissons en une dizaine de minutes et ne détruit nullement les parasites dans ce laps de temps. La solution à $2 / 1.000$ fait mourir les alevins en 2 ou 3 minutes sans immobiliser les parasites.

XIV. Action de l'air sec et du soleil sur les " poux des poissons ". - Dans une première série d'expériences des Ichthyophtirius isolés de leur hôte sont mis dans deux tubes de verre contenant de l'eau ordinaire. Un tube est laissé comme témoin au laboratoire à $20^{\circ}$; l'autre est exposé en plein soleil $\left(36^{\circ}\right)$ pendant 2 heures. Après ce temps le tube ensoleillé ne renferme plus que des parasites immobiles alors que dans le témoin tous les infusoires sont vivants.

Pour l'autre série d'expérimentation, des alevins malades sont sortis de l'eau et exposés à l'air sec et au soleil. Du 20 juillet à 16 heures au 21 juillet à 11 heures du matin, soit durant 19 heures, quelques truites parasitées sont abandonnées en dehors du laboratoire à l'air sec et au soleil, aux températures 'de $27^{\circ}$ à 9 heures et $31^{\circ}$ à 11 heures. Le grattage de la peau des cadavres est mis dans des verres de montre remplis d'eau et reportés au laboratoire. Une demiheure comme 4 heures plus tard le contenu des verres de montre ne renferme plus que des Ichthyophtirius complètement immobiles.

Le 21 juillet à 8 heures 45 du matin, plusieurs poissons malades sont exposés en plein soleil $\left(27^{\circ}\right.$ à 9 heures, $31^{\circ}$ à 11 heures, $34^{\circ}$ à 15 heures 30 ). A 11 heures du matin, soit après 2 heures 15 d'insolation, le râclage des téguments parasités contient encore des lchthyophtirius bien mobiles, tandis que les grattages de peau effectués à 15 heures 15 , soit après 6 heures et demie d'insolation, ne renferment plus un seul parasite vivant.

Conclusions Expérimentales. - De ces différentes expériences on peut déduire que :

$1^{\circ}$ De par ses branchies très fragiles, la jeune truite est excessivement sensible aux réactifs chimiques qui la font périr longtemps avant d'atteindre le parasite protégé par un abondant mucus. II 
semble bien difficile de prévoir un produit chimique à la fois inoffensif pour le délicat appareil respiratoire du poisson et nocif pour un parasite aussi résistant. Dans la lutte contre l'épidémie, seuls les procédés mécaniques et physiques, frottement, eau très courante et froide, peuvent donner des résultats positifs.

$2^{\circ}$ L'Ichthyophtirius a une très grande vitalité, les données expérimentales le prouvent surabondamment. Si l'on se place au point de vue pratique, on aperçoit des procédés commodes de désinfection des bassins et des objets contaminés. C'est, d'une part, la dessiccation à l'air, l'insolation, l'eau de chaux pour les étangs asséchés et pour l'outillage ; d'autre part, le formol à 1 pour cent ou le lait de chaux pour la désinfection rapide des mains du pisciculteur ou des récipients de l'exploitation.

\section{Discussion des observations et des résultats Conclusions générales}

Les épidémies dues à Ichthyophtirius sévissent surtout pendant la belle saison, printemps et été. Pourquoi pendant cette période de l'année et non pas en hiver?

On sait que d'une façon générale et dans certaines limites, la chaleur est un facteur important de la rapidité de la multiplication cellulaire. Il n'y a donc rien de surprenant que la température plus élevée de l'été favorise le développement du parasite. Mais la truite supporte mal le séjour dans les eaux tièdes et insuffisamment renouvelées, elle s'y affaiblit et ralentit ses mouvements. Nous avons en présence des parasites vivant dans les conditions les plus favorables pour leur évolution des hôtes à la limite de résistance. L'issue de ce combat inégal et inévitable est prévue en faveur de l'Ichthyophtirius. Ce simple rapprochement de la biologie des deux êtres nous indique pourquoi l'épidémie que j'ai relatée a débuté dans un lot de truites de rivière d'origine danoise, élevées dans une eau peu renouvelée à $18^{\circ}-19^{\circ}$, température incompatible pour la vie normale de ces poissons. Nous nous expliquons aussi pourquoi les autres espèces de truites, plus robustes, supportant mieux cette température élevée ont résisté davantage au parasite.

On ne saurait trop insister sur les faits suivants :

$1^{\circ}$ Le poisson meurt par asphyxie provoquée par les Ichthyophtirius, recouvrant les branchies et empêchant l'hématose du sang.

$2^{\circ}$ Après la mort de l'hôte, le parasite le quitte, mais ne périclite pas. Il reste en suspension dans l'eau, et s'il n'est pas entraìné par 
le courant, il tombe au fond du vivier pour continuer à vivre. C'est une constatation que j'ai faite à plusieurs reprises dans des bacs renfermant de nombreuses truites malades.

Comme les produits chimiques ne peuvent débarrasser efficacement le poisson de ses parasites, dans la lutte contre l'épidémie, les mesures prophylactiques ou curatives à prendre consisteront donc avant tout, d'une part à affaiblir le parasite, à ralentir, ou à annihiler son développement, et d'autre part, à augmenter la résistance et la vivacité du poisson.

L'abaissement de la température de l'eau fournie en grande abondance et en courant rapide, diminue la prolifération des parasites tout en les entrainant en idehors des viviers. En même temps, la truite, vivant dans une eau plus froide et un milieu plus aéré reprend une allure plus vive, qui lui permet de se débarrasser par frottement des quelques Ichthyophtirius que le hasard aurait fixé à ses téguments. Le mode d'alimentation en eau et la disposition des étangs ont également une grande importance. Pendant la période estivale, dans les points morts, l'eau ne se renouvelant pas, élève sa température et favorise le développement et l'accumulation d'ennemis dangereux que des mollusques aquatiques ou d'autres vecteurs peuvent colporter dans les régions saines. Dans un établissement de pisciculture modèle, l'origine des poissons est contrôlée, et on élève seulement des espèces robustes et rustiques, pouvant résister aux fluctuations ide température de la région.

Enfin, en période d'épidémie, les mesures à prendre sont les suivantes : soumettre les malades à un fort courant d'eau fraîche, vider les viviers et les laisser sans eau pendant quelques jours, le soleil et l'air détruisant un grand nombre de parasites. Puis il sera utile de projeter de la chaux vive dans les bassins et quelque temps après, de faire rentrer l'eau. Ce n'est que plus tard, lorsque cette eau de chaux se sera carbonatée en partie, qu'elle pourra sans inconvénient être rejetée par petites portions à la fois dans le cours d'eau voisin.

Les instruments, les outils, les récipients de l'établissement seront exposés à l'air sec et au soleil ou passés dans un lait de chaux. Quant aux mains des travailleurs, un lait de chaux ou du formol à un pour cent seront suffisants pour les désinfecter.

\section{RÉSUMÉ}

L'épidémie a sévi dans un établissement de pisciculture de l'Est de la France, pendant les fortes chaleurs de l'été 1923 . Elle a débuté sur des alevins de truites de rivière (Samo fario), d'origine danoise 
qui supportent mal les températures supérieures à $18^{\circ}-19^{\circ}$. Les poissons meurent par asphyxie provoquée par les Ichthyophtirius qui recouvrent les branchies, et non par suite des lésions de la peau.

Différents produits chrmiques utilisés (permanganate de potassium, chlorure de sodium, chlorure mercurique, eau de Javelle, formol, toluène, chloral hydraté, camphre, pyridine, créosote, eau oxygéné, thymol) ont été inefficaces dans la lutte directe contre l'épizootie : les poissons malades périssent avant que les parasites protégés par un abondant mucus ne soient atteints.

Seuls, les procédés physiques et mécaniques (frottement, circulation d'eau courante et fraîche) ont permis d'enrayer la maladie.

Les conclusions de l'auteur sont les suivantes : $1^{\circ}$ La température anormale de l'eau insuffisamment renouvelée, tout en affaiblissant la résistance des truites, favorise le développement rapide des parasites qui se fixent sur les branchies de l'hôte et le font mourir par asphyxie ;

$2^{\circ}$ Les mesures prophylactiques et curatives à prendre consistent essentiellement à établir une circulation intense d'eau fraîche dans les étangs ; à assécher, insoler, chauler les bassins contaminés et à désinfecter les mains du pisciculteur et l'outillage de l'exploitation dans un lait de chaux ou dans du formol à un pour cent.

\section{Bibliographie (1)}

Bưcshкiel (A.-L.). - Beiträge zur Kenntnis des Ichthyophtirius multifiliis Fouquet. Archiv für Protistenkunde, XXI, 1911.

Doflein (F.). - Lehrbuch der Protozoenkunde. II. Auflage der "Protozoen als Parasiten und Krankheitserreger 》, 1916.

Fovquet (D.). - Note sur une espèce d'infusoires parasites des poissons d'eau douce. Archives de Zoologie expérimentale el générale, V, 1876.

HOFER (B.). - Hañdbuch der Fischkrankheiten. München, 1904.

Kerbert (C.). - Chromatophagus parasiticus nov. gen. nov. spec. Niderl. Tijdsch. v. d. dierk. Vereen. Amsterdam, Jahrg. 5, 1882.

Neresheimer (E.). - Der Zeugungskreis des Ichthyophtirius. Stuttgart E. Schweizerbart'sche Verlagsbuchhandlung E. Nägele, 1908.

Raveret-Wattel (C.). - Atlas de poche des poissons d'eau donce de France, Suisse et Belgique, Paris.

StiLes (C.-W.). - Report upon a parasitic protozoan observed on fish in the aquarium. Bull. of the U. S. fish commission, V. 13, 1893.

Weltner (W.). - Die Ichthyophtirius-Krankheit. Blätter $f$. Aquarien u. Terrarienliunde, $\mathrm{V}, 6,1895$.

Institut de Zoologie et de Biologie générale de la Faculté des Sciences de Strasbourg.

(1) Pour la bibliographie plus complète consulter: Dofleis, Lehrbuch der Protozoenkunde, 1916, et BuschkiEL, Archiv für Protistenkunde, XXI, 1911. 